\title{
Effects of Brood Manipulation Costs on Optimal Sex Allocation in Social Hymenoptera
}

\author{
Max Reuter, ${ }^{\star}$ Ken R. Helms, ${ }^{\dagger}$ Laurent Lehmann, ${ }^{\ddagger}$ and Laurent Keller ${ }^{\S}$
}

Department of Ecology and Evolution, University of Lausanne, Bâtiment de Biologie, 1015 Lausanne, Switzerland

Submitted July 14, 2003; Accepted April 5, 2004;

Electronically published July 26, 2004

ABstract: In eusocial Hymenoptera, queens and workers are in conflict over optimal sex allocation. Sex ratio theory, while generating predictions on the extent of this conflict under a wide range of conditions, has largely neglected the fact that worker control of investment almost certainly requires the manipulation of brood sex ratio. This manipulation is likely to incur costs, for example, if workers eliminate male larvae or rear more females as sexuals rather than workers. In this article, we present a model of sex ratio evolution under worker control that incorporates costs of brood manipulation. We assume cost to be a continuous, increasing function of the magnitude of sex ratio manipulation. We demonstrate that costs counterselect sex ratio biasing, which leads to less female-biased population sex ratios than expected on the basis of relatedness asymmetry. Furthermore, differently shaped cost functions lead to different equilibria of manipulation at the colony level. While linear and accelerating cost functions generate monomorphic equilibria, decelerating costs lead to a process of evolutionary branching and hence split sex ratios.

Keywords: kin selection, conflict, model, evolutionary branching.

Queen-worker conflict over sex allocation has been an important area of research on social insects over the past 25 years. As first outlined by Trivers and Hare (1976),

\footnotetext{
* Corresponding author. Present address: Department of Biology, University College London, Galton Laboratory, 4 Stephenson Way, London NW1 2HE, United Kingdom; e-mail: m.reuter@ucl.ac.uk.

+ Present address: Department of Entomology, Texas A\&M University, College Station, Texas 77843-2475; e-mail: khelms@neo.tamu.edu.

${ }^{\ddagger}$ Present address: Department of Biological and Environmental Science, University of Helsinki, P.O. Box 65, 00014 Helsinki, Finland; e-mail: llehmann@mappi.helsinki.fi.

` E-mail: laurent.keller@ie-zea.unil.ch.
}

Am. Nat. 2004. Vol. 164, pp. E73-E82. (c) 2004 by The University of Chicago. 0003-0147/2004/16403-40005\$15.00. All rights reserved. queens and workers in a hymenopteran colony differ in their optimal sex allocation. This difference is caused by relatedness asymmetries arising from haplodiploidy, the hymenopteran system of sex determination. In haplodiploid species, females develop from fertilized (diploid) eggs, whereas males develop from unfertilized (haploid) eggs. As a consequence, in a colony headed by one singly mated queen, workers are three times more related to female sexuals (their sisters; life-for-life relatedness $r=$ 0.75 ) than to male sexuals (their brothers; life-for-life relatedness $r=0.25$ ). Corresponding to this asymmetry in relatedness, equilibrium sex allocation under worker control is female biased, with three times more resources invested in females than in males. Queens, in contrast, are equally related to sons and daughters, and their equilibrium sex ratio is therefore an equal investment in the two sexes.

Since Trivers and Hare, hymenopteran sex ratio theory has been greatly developed. Predictions of optimal sex allocation under queen and worker control have been generated to include a variety of genetic structures of colonies and populations (for reviews, see Bourke and Franks 1995; Crozier and Pamilo 1996). However, almost all theoretical work has assumed that there are no costs associated with sex ratio manipulation. This assumption is likely to be wrong, especially in the case of workers. In order to alter sex allocation, workers have to manipulate the primary egg sex ratio, which is controlled by the queen. There are two likely mechanisms of sex ratio manipulation. First, workers can eliminate male eggs or larvae to increase the relative investment in females. This mechanism has been found to be used by workers of the ant Formica exsecta (Sundström et al. 1996). However, in this species the energy already invested in eliminated males cannot be completely recovered, meaning that sex ratio adjustment is likely to result in a loss of resources (Chapuisat et al. 1997). Additional costs may arise if the time span between elimination of males and the end of the reproductive season is too short for all replacement females to complete development or if the sex of larvae is identified with error. A second mechanism allowing workers to adjust sex al- 
location is to alter the proportion of females developing into queens and workers (i.e., influence caste determination). In many species, the quantity and/or quality of food provided during larval development appears important in determining whether females develop into workers or gynes (female sexuals). Workers can therefore influence sex allocation by influencing the process of caste determination and increasing the proportion of females reared as sexuals. This mechanism of sex ratio biasing has been found to be used by workers of the ant Leptothorax acervorum (Hammond et al. 2002). Sex ratio manipulation through the biasing of female caste determination should also be associated with costs because raising more females as gynes reduces investment in new workers, thereby compromising colony growth and overall productivity (Reuter and Keller 2001).

In this article we investigate how costs inherent to the workers' sex ratio manipulation influence the evolution of sex allocation in eusocial Hymenoptera. In the absence of data on the relation between the degree of sex ratio manipulation and associated costs, we implement the cost function in a general form that can take a linear, accelerating, or decelerating shape. Interestingly, we found that the type of cost function is important. Linear and accelerating costs select for a monomorphic reduction of sex ratio manipulation; that is, all colonies produce a lower proportion of females than predicted from relatedness asymmetry in the absence of cost. In contrast, decelerating costs lead to a process of evolutionary branching and hence split sex ratios, with workers of some colonies biasing sex ratio up to a complete female production while workers of other colonies cease to manipulate sex allocation altogether.

\section{The Model}

We consider a very large (infinite) population of colonies composed of queens and workers. Throughout this article we will assume the presence of one singly mated queen per colony. (It is important to note that as long as kin structure does not vary between colonies or workers do not react to variations in colony kin structure, similar results would be obtained with any number of queens and/ or mating frequency. The reason is that fitness is a linear function of degrees of relatedness and selection pressures thus remain proportional with changes in kin structure.) Colonies produce one batch of sexuals. For this purpose, queens lay sexual-destined eggs in a primary sex ratio $p$, defined either as the proportion of female eggs (if males and females are equally costly to produce) or as the proportion of female eggs that would result in an sex investment ratio of $p$ if raised without manipulation (if males and females are not equally costly to produce). The pri- mary sex ratio $p$ is assumed to be the same in all colonies. In numerical calculations, we assume its value to be equal to the queens' equilibrium sex ratio, $p=0.5$ (assuming that a different value of $p \in[0,0.75]$ does not change our results qualitatively). Workers of colony $i$ manipulate the primary sex ratio and increase the proportional investment in females by an amount $m_{i} \in[0,1-p]$, resulting in a secondary sex ratio of $s_{i}=p+m_{i}$.

The manipulation of sex ratio is assumed to entail a cost by reducing the productivity of colony $i, P_{i}$, by a proportion $\gamma\left(m_{i}\right)$, such that $P_{i}=1-\gamma\left(m_{i}\right)$. The cost function $\gamma$ is of the general form $\gamma\left(m_{i}\right)=b\left[m_{i} /(1-p)\right]^{a}$. In this function, $b$ determines the cost required to manipulate sex allocation up to a complete female bias $\left(m_{i}=1-p\right)$ and thus defines the overall slope of the cost function. The parameter $a$ determines the curvature of the cost function. The function is linear when $a=1$, accelerating when $a>1$, and decelerating when $a<1$.

The sexuals produced are assumed to mate randomly in the population, and the mating success of a given sex is proportional to the inverse of the population mean investment in that sex. Following Pamilo (1991) and Boomsma and Grafen (1991), we express the fitness that workers of colony $i$ gain through sexual offspring of one sex as the product of mating success, reproductive value $\left(\nu_{\mathrm{m}}\right.$ and $\left.\nu_{\mathrm{f}}\right)$, and the workers' regression relatedness to individuals of that sex $\left(g_{\mathrm{m}}\right.$ and $\left.g_{\mathrm{f}}\right)$. The total worker fitness $V_{i}$ is given by the sum of fitness gained through males and females, weighted by colony investment in the two sexes and colony productivity:

$$
V_{i}=\nu_{\mathrm{m}} g_{\mathrm{m}} \frac{1-s_{i}}{1-s} P_{i}+\nu_{\mathrm{f}} g_{\mathrm{f}} \frac{s_{i}}{s} P_{i}
$$

where $s$ is the mean investment in females in the population. The selective pressures acting on the workers' sex ratio manipulation can be analyzed by taking the derivative of fitness with respect to manipulation strategy

$$
\frac{d V_{i}}{d m_{i}}=\nu_{\mathrm{m}} g_{\mathrm{m}} \frac{d\left(\frac{1-s_{i}}{1-s} P_{i}\right)}{d m_{i}}+\nu_{\mathrm{f}} g_{\mathrm{f}} \frac{d\left(\frac{s_{i}}{s} P_{i}\right)}{d m_{i}}
$$

which is equivalent to the inclusive fitness effect in Taylor (1996; eq. [4.1]). Setting this expression equal to 0 and substituting $m_{i}=m=m^{*}$ gives the equilibrium condition for sex ratio manipulation:

$$
\gamma^{\prime}\left(\nu_{\mathrm{m}} g_{\mathrm{m}}+\nu_{\mathrm{f}} g_{\mathrm{f}}\right)=s^{\prime}(1-\gamma)\left(\nu_{\mathrm{f}} g_{\mathrm{f}} \frac{1}{s}-\nu_{\mathrm{m}} g_{\mathrm{m}} \frac{1}{1-s}\right)
$$

where $s^{\prime}$ and $\gamma^{\prime}$ denote derivatives of $s$ and $\gamma$ with respect 
to $m_{i}$. Equation (3) is arranged so as to emphasize the selective forces acting on worker sex ratio manipulation. The left-hand side corresponds to selection against sex ratio manipulation that is proportional to $\gamma^{\prime}$, the change in colony productivity resulting from a small change in worker manipulation $m_{i}$. Depending on the form of the cost function, selection resulting from decreased colony productivity can be independent of manipulation $m_{i}$ (linear costs), increase with $m_{i}$ (accelerating costs), or decrease with $m_{i}$ (decelerating costs). The right-hand side assembles the selective force in favor of sex ratio manipulation, which is proportional to the change in sex ratio that results from a small change in manipulation $\left(s^{\prime}\right)$ and the genetic benefit of manipulation, the difference in the workers' relatedness to females and males weighted by their mating success. The strength of selection in favor of sex ratio manipulation decreases with increasing female bias in population sex ratio and vanishes at the workers' sex ratio equilibrium as predicted on the basis of relatedness asymmetry.

Under the influence of the two opposing selective forces, worker manipulation will evolve toward an equilibrium value $m^{*}$ at which selection for and against manipulation is balanced. If the sex ratio is less female biased than the workers' optimum, selection in favor of manipulation will be stronger than counterselection exerted by costs, and manipulation will increase. If the sex ratio is more female biased than the optimum, manipulation will be reduced because productivity costs select more strongly against manipulation than genetic benefits select in its favor. In the case where sex ratio manipulation has no cost $(b=0)$, the balance between the selective forces is reached at the sex ratio where genetic benefits of further bias are 0 , that is, at the sex ratio predicted by Trivers and Hare:

$$
m^{*}=\frac{\nu_{\mathrm{f}} g_{\mathrm{f}}}{\nu_{\mathrm{f}} g_{\mathrm{f}}+\nu_{\mathrm{m}} g_{\mathrm{m}}}-p
$$

Thus, in the absence of cost, workers will manipulate sex allocation up to a value of $\nu_{\mathrm{f}} g_{\mathrm{f}} /\left(\nu_{\mathrm{f}} g_{\mathrm{f}}+\nu_{\mathrm{m}} g_{\mathrm{m}}\right)$, that is, up to a secondary sex ratio of $3: 1$ in colonies headed by one once-mated queen.

When sex ratio manipulation incurs costs, the equilibrium sex ratio will deviate from $3: 1$. The sex ratio equilibrium reached with linear and accelerating costs is qualitatively different from that with decelerating cost. The two cases will therefore be treated separately in the following sections.

\section{Linear and Accelerating Costs}

With linear and accelerating costs $(a \geq 1)$ we can identify a single monomorphic equilibrium $m^{*}$. This equilibrium is a continuously stable strategy (CSS; Eshel and Motro
1981; Christiansen 1991; Taylor 1996). It fulfills the conditions of local mutant stability commonly used to characterize an evolutionarily stable strategy (ESS), in that

$$
\begin{aligned}
& \left.\frac{\partial V_{i}}{\partial m_{i}}\right|_{m_{i}=m=m^{*}}=0, \\
& \left.\frac{\partial^{2} V_{i}}{\partial m_{i}^{2}}\right|_{m_{i}=m=m^{*}}<0 .
\end{aligned}
$$

These two conditions indicate that $m^{*}$ is a fitness maximum and that populations fixed for the equilibrium strategy cannot be invaded by any mutant whose strategy deviates slightly from $m^{*}$. In addition, the equilibrium fulfils the condition for population stability required for a CSS:

$$
\frac{\partial}{\partial m_{\mathrm{d}}}\left(\left.\frac{\partial V_{i}}{\partial m_{i}}\right|_{m_{i}=m_{\mathrm{d}}}\right)_{m_{\mathrm{d}}=m^{*}}<0 .
$$

The last condition states that a population whose mean strategy $m_{\mathrm{d}}$ deviates from the stable equilibrium $m^{*}$ will evolve toward $m^{*}$ because individual strategies $m_{i}$ closer to $m^{*}$ are favored by selection. This property makes the CSS stronger than the ESS because populations will not only be maintained at the equilibrium strategy once they have attained it, but they will also move toward the equilibrium even if starting from a different strategy or when perturbed.

The numerical value of the evolutionary stable sex ratio manipulation depends on the slope $b$ and the curvature $a$ of the cost function. Figure $1 A$ summarizes the results of numerical calculations with linear costs $(a=1)$ as a function of the slope $b$. As expected, the evolutionary stable sex ratio manipulation decreases with increasing slope of the cost function, indicating that workers will bias sex allocation less if manipulation has stronger negative effects on colony productivity. Manipulation costs exert an important selective pressure on sex allocation. A cost that would cause a $10 \%$ reduction in productivity when altering the sex allocation to $3: 1(b=0.2)$, for example, shifts the optimal sex allocation to less than $2: 1$. The importance of colony productivity for worker fitness is further demonstrated by the fact that the actual cost paid by colonies at equilibrium is low (fig. $1 B$ ). This indicates that workers are never selected to accept large decreases in colony productivity in order to attain a more female-biased sex allocation.

Accelerating costs $(a>1)$ lead to an increase in the evolutionary stable sex ratio manipulation, as compared with 

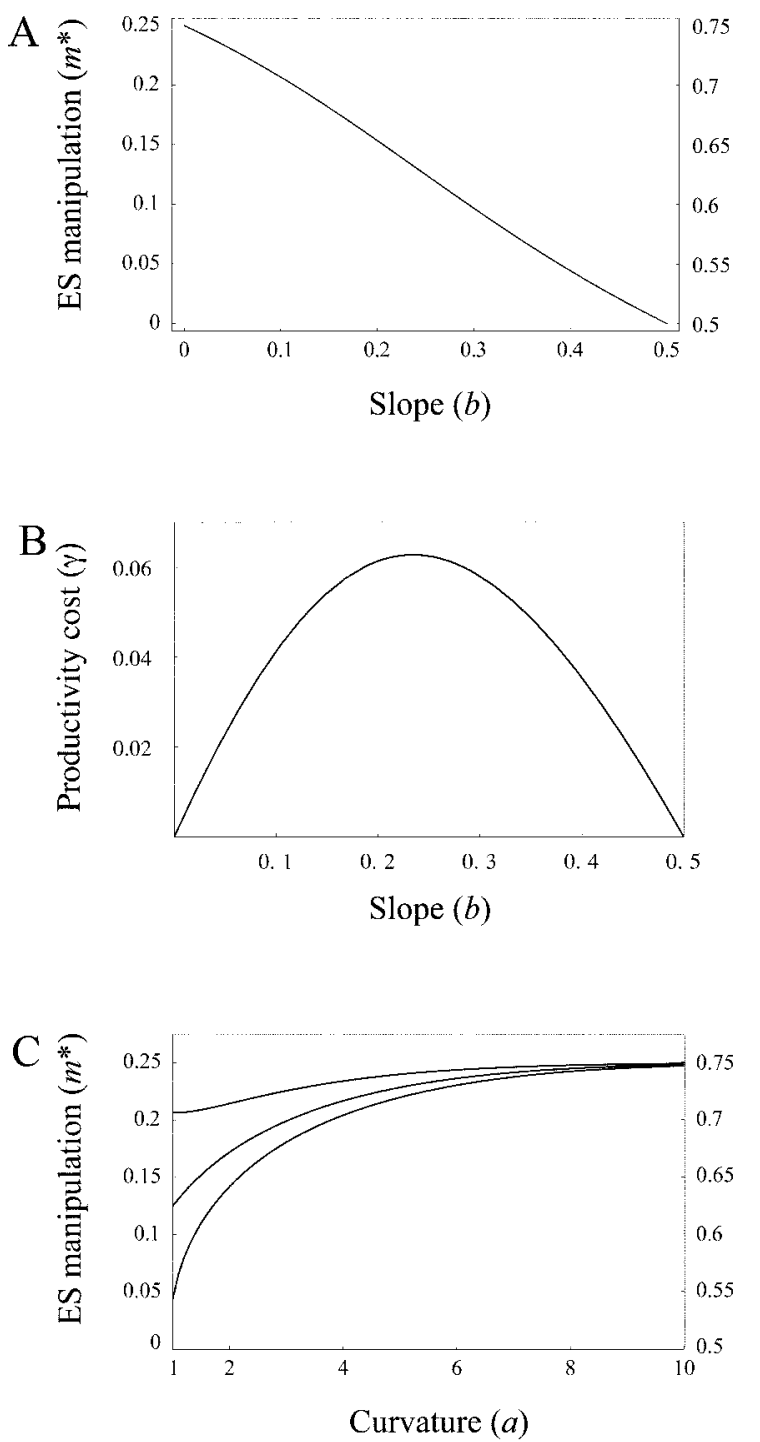

Figure 1: Evolution of sex ratio manipulation with linear and accelerating costs. A, Evolutionary stable (ES) sex ratio manipulation $\left(m^{*}\right)$ and the resulting sex ratio $(s)$ as a function of the slope $b$ of a linear cost function. $B$, Productivity cost at equilibrium as a function of the slope $b$ of a linear cost function. $C$, Evolutionary stable sex ratio manipulation $\left(m^{*}\right)$ and the resulting sex ratio $(s)$ as a function of the curvature $a$ of an accelerating cost function. Results are given for three slopes (from top: $b=0.1$, $b=0.25, b=0.4$ ).

linear costs (fig. 1C). The reason is that concave curvature reduces the slope of the cost function for low and intermediate values of manipulation while increasing it for high values. For very high values of $a$, the cost function $\gamma(m)$ is almost flat for $m<0.5$ and then increases very steeply to reach a cost of $b$ for $m=0.5$. As long as manipulation is intermediate, curvature thus effectively reduces the costs of sex ratio manipulation and allows workers to attain more female-biased sex ratios.

\section{Decelerating Costs}

The evolution of sex ratio manipulation with decelerating costs is more complicated than with linear or accelerating costs because the dynamics depend on the values of $a$ and $b$. Two cases are possible depending on whether $\{a, b\}$ falls into region 1 or 2 of the parameter space depicted in figure 2. To understand the difference between the two cases, it is helpful to represent the evolutionary dynamics by pairwise invisibility plots (fig. 3). These plots indicate which mutant strategies $m^{\prime}$ can invade populations with resident strategies $m$. Pairs $\left\{m^{\prime}, m\right\}$ for which the mutant has a higher fitness than the resident lie in areas of the plot marked with a plus sign. Pairs $\left\{m^{\prime}, m\right\}$ for which the mutant has a lower fitness than the resident lie in areas marked with a minus sign. The lines separating the areas follow isoclines of equal mutant and resident fitness, and line intersections indicate equilibria, the stability of which depends on the angle of intersection as well as the sign of the four areas surrounding the point of intersection (Geritz et al. 1998).

Figure $3 A$ shows the pairwise invasibility plot for points $\{a, b\}$ in $R_{1}$. There are two evolutionary equilibria, $m_{0}^{*}=0$ and an internal equilibrium $m_{\mathrm{int}}^{*}$. Both are sep-

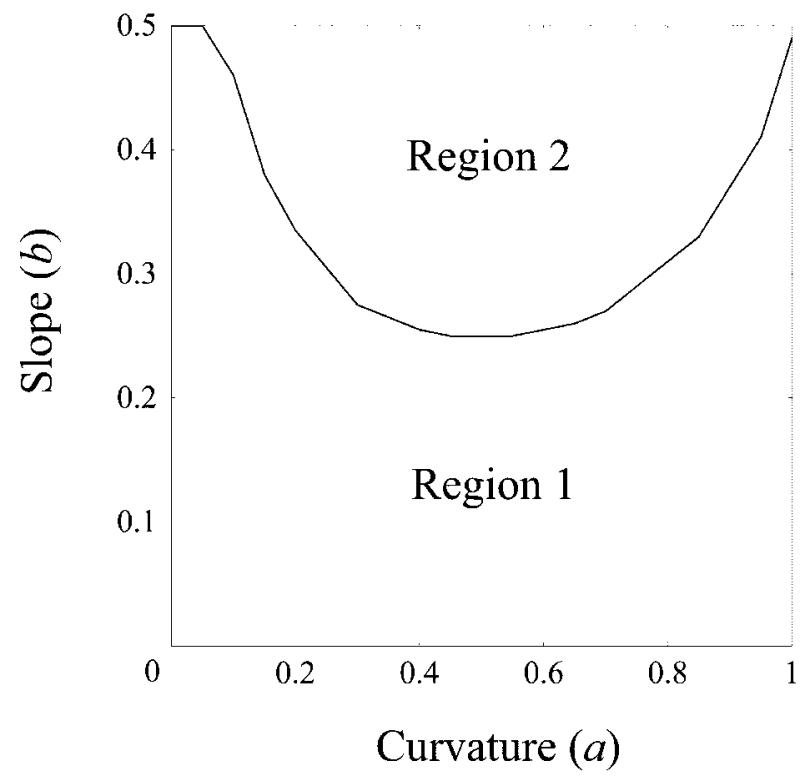

Figure 2: Regions of the parameter space of slope $b$ and curvature $a$ differing in invasion conditions. The pairwise invasibility plots for pairs $\{a, b\}$ falling into regions 1 and 2 are given in figure $3 A$ and $3 B$, respectively. 

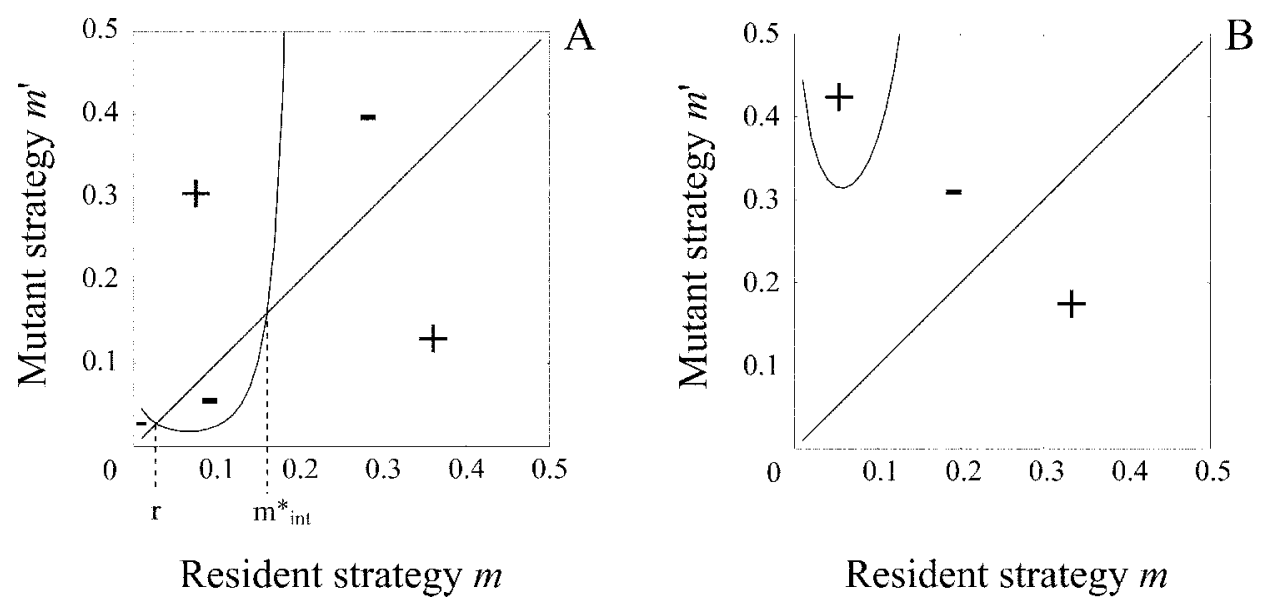

Figure 3: Pairwise invasibility plots for parameters $\{a, b\}$ falling into region $1(A)$ and region $2(B)$ in figure 2 . Areas marked with a plus sign indicate combinations of mutant strategies $m^{\prime}$ and resident strategies $m$ for which mutants have a higher fitness than residents and can invade.

arated by a point $r$ that is a repeller for both individuals (eq. [6] not fulfilled) and populations (eq. [7] not fulfilled). The equilibrium $m_{0}^{*}=0$ is stable only at a very local scale. As shown in figure $3 \mathrm{~A}$, in a resident population at $m_{0}^{*}$, only mutant strategies deviating very slightly from the resident (i.e., below $r$ ) are counterselected while others can invade. Thus, $m_{0}^{*}$ is not a stable equilibrium if mutations with large effects can occur. By contrast, the internal equilibrium $m_{\mathrm{int}}^{*}$ is stable because it fulfills the condition of population stability (eq. [7]), indicating that selection will maintain the mean population strategy at $m_{\mathrm{int}}^{*}$. Interestingly, however, the equilibrium is not an ESS at the individual level (eq. [6] not fulfilled). Situations combining population stability with mutant instability are known to lead to a process of evolutionary branching (Taylor 1996; Geritz et al. 1998). Populations will thus evolve toward the equilibrium, but once they reach it, disruptive selection acts on individual strategies leading to the fixation of two extreme strategies, which in our case are $m_{\text {none }}^{*}=0$ (no manipulation) and $m_{\text {all }}^{*}=1-p$ (manipulation to complete female production).

For points $\{a, b\}$ in $R_{2}$, the fitness isoclines in the pairwise invasibility plot do not intersect (fig. 3B). The derivative $\partial V_{i} /\left.\partial m_{i}\right|_{m_{i}=m}$ is negative for all $m \in[0,1-p]$, and the only possible monomorphic equilibrium is $m^{*}=0$. However, in populations near this equilibrium, invasion is possible by individual strategies with levels of sex ratio manipulation high enough to fall in the region marked with a plus sign. Thus, if mutations with large effects occur, populations will again evolve toward a dimorphic equilibrium with $m_{\text {none }}=0$ and $m_{\text {all }}=1-p$.

The frequencies of the two manipulation strategies $m_{\text {none }}$ and $m_{\text {all }}$ depend on the shape of the cost function.
To predict these frequencies, we can investigate the evolution of a trait $q_{i}$ (with population mean $q$ ) defined as the frequency with which workers of colony $i$ apply strategy $m_{\text {none }}$ (no manipulation) as opposed to $m_{\text {all }}$ (complete female production, applied with frequency $1-q)$. The equilibrium frequency $q^{*}$ can be obtained by maximizing the equation

$$
\begin{aligned}
V_{i}= & q_{i}\left(\nu_{\mathrm{f}} g_{\mathrm{f}} \frac{s_{\text {none }}}{s} \frac{1}{P}+\nu_{\mathrm{m}} g_{\mathrm{m}} \frac{1-s_{\text {none }}}{1-s} \frac{1}{P}\right) \\
& +\left(1-q_{i}\right)\left(\nu_{\mathrm{f}} g_{\mathrm{f}} \frac{1}{s} \frac{P_{\text {all }}}{P}\right)
\end{aligned}
$$

for $q_{i}$, with $q_{i}=q=q^{*}, P_{\text {all }}=\left[1-\gamma\left(m_{\text {all }}\right)\right], P=q+$ $(1-q) P_{\text {all }}$, and $s=\left[q s_{\text {none }}+(1-q) P_{\text {all }}\right] / P$. The equilibrium frequency $q^{*}$ is given by

$$
q^{*}=\frac{\nu_{\mathrm{m}} g_{\mathrm{m}}(1-b)}{\left(\nu_{\mathrm{m}} g_{\mathrm{m}}+\nu_{\mathrm{f}} g_{\mathrm{f}}\right)(1-p-b)}
$$

which can be shown to be a CSS. Because manipulation is either absent $\left(m_{\text {none }}=0\right)$ or complete $\left(m_{\text {all }}=1-p\right)$, $q^{*}$ is independent of the curvature $a$ of the cost function. In contrast, the maximum cost of sex ratio manipulation (b) has a strong influence on the equilibrium strategy. With increasing slope $b$ of the cost function (i.e., increasing costs of producing all-female broods), the evolutionary stable frequency of nonmanipulating colonies increases (fig. 4A). Concurrently, the population sex ratio becomes less female biased (fig. 4B). 

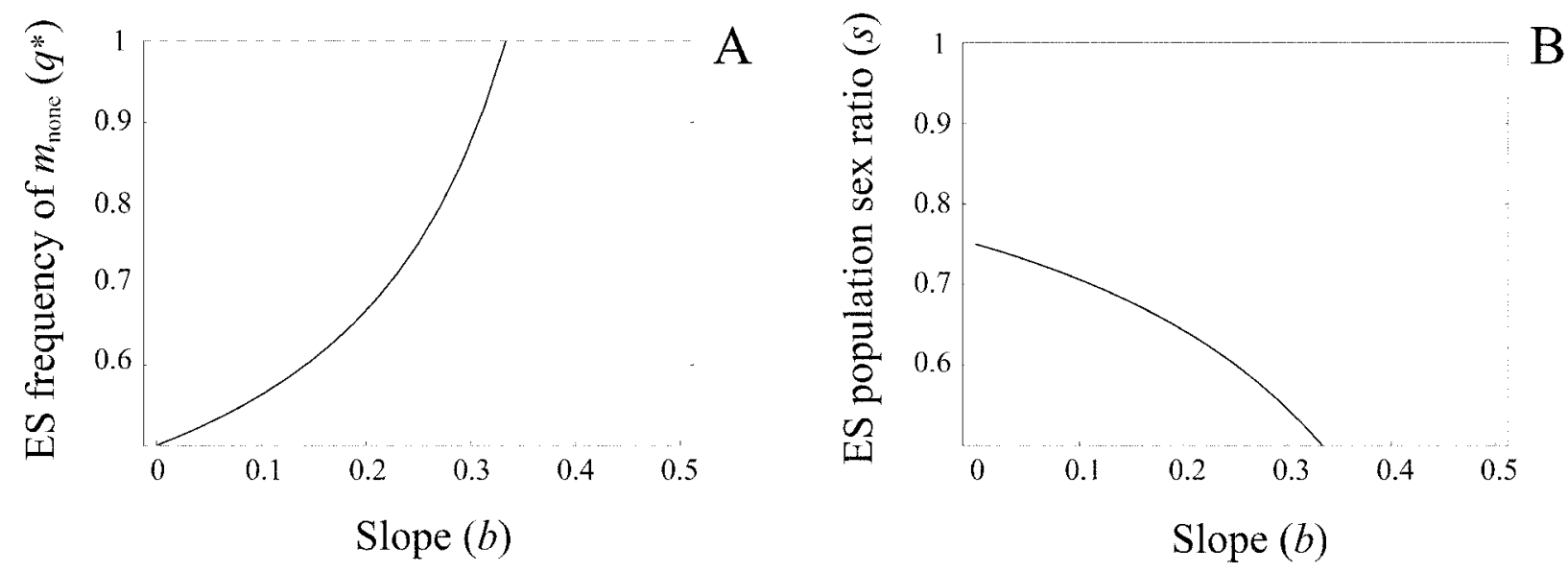

Figure 4: Evolutionary stable frequency of nonmanipulating workers $\left(A ; q^{*}\right)$ and the resulting population sex ratio $(B$; $s)$ as a function of the slope of the decelerating cost function $(b)$.

\section{Selection on Queen Response}

In the previous sections we have shown that, under a wide range of conditions, workers are selected to increase female investment beyond the primary ratio of $p=1 / 2$. This raises the issue of whether, in the face of worker manipulation, queens benefit to bias the primary sex ratio in opposition to worker manipulation, or alternatively, whether they would benefit to lay eggs in the workers' equilibrium sex ratio so as to avoid the productivity costs associated with the workers' manipulation. This question is important because actual conflict involving worker sex ratio manipulation will occur only if queens produce a primary sex ratio different from the workers' optimum.

To investigate possible queen responses to worker manipulation, we can analyze the fitness of a mutant queen phenotype producing a primary sex ratio of $p_{\mathrm{m}}$ in a population where queens produce a $p=1 / 2$ primary sex ratio and workers manipulate this sex ratio by $m^{*}$. The direction of selection on the mutant queen is given by the sign of the derivative of queen fitness $V_{\mathrm{Q}}$ with respect to $p_{\mathrm{m}}$. Using equation (1) with $s_{i}=p_{\mathrm{m}}+m^{*}$ and $s=p+m^{*}$ and assuming monogyny and single-queen mating $\left(\nu_{\mathrm{f}} g_{\mathrm{f}}=1 / 2\right.$, $\left.\nu_{\mathrm{m}} g_{\mathrm{m}}=1 / 2\right)$, we obtain

$$
\begin{aligned}
\frac{d V_{\mathrm{Q}}}{d p_{\mathrm{m}}} & =\frac{\nu_{\mathrm{f}} g_{\mathrm{f}}}{p+m^{*}}-\frac{\nu_{\mathrm{m}} g_{\mathrm{m}}}{1-p+m^{*}} \\
& =\frac{\frac{1}{2}-\left(p+m^{*}\right)}{\left(p+m^{*}\right)-\left(p+m^{*}\right)^{2}} .
\end{aligned}
$$

For secondary sex ratios between 0 and 1 , the denominator of the right-hand ratio is always $>0$ and the direction of selection is given by the sign of the numerator. The numerator is negative if $p+m^{*}>1 / 2$, implying that queens are selected to reduce the proportion of females in the primary sex ratio whenever the average female investment in the population exceeds their equilibrium sex allocation of $s=1 / 2$. This indicates that queens are not selected to concede to the workers' sex ratio manipulation. Rather, the queen response tends to increase the difference between primary sex ratio and the workers' optimum and hence augments the scope for actual conflict between the two parties.

\section{Discussion}

Our model shows that the costs associated with sex ratio manipulation affect the evolution of sex allocation at both the population and colony levels. When manipulation incurs costs, the workers' genetic benefit of biasing sex ratio toward more closely related females is partly offset by the reduction in overall sexual production and workers are selected to reduce the level of brood manipulation. Our model therefore predicts that if brood manipulation has costs, the equilibrium sex allocation should be less femalebiased than predicted by models based on relatedness asymmetry alone (figs. 1, 4).

At the population level our model shows that female bias decreases concomitantly with increased costs of manipulation. This prediction has two important implications. First, the population sex ratio predicted by relatedness asymmetry will be attained only in the absence of costs of manipulation. This is the situation envisioned by Trivers and Hare when assuming worker control of sex allocation. However, this situation is probably rarely, if 
ever, fulfilled in social Hymenoptera because the known mechanisms of sex ratio manipulation are costly. Costs of sex ratio manipulation may thus account at least in part for the observation that sex allocation across monogynous ant species is significantly less female biased than $3: 1$ (Boomsma 1989; Pamilo 1990). Other potential causes leading to a less than $3: 1$ female-biased sex investment ratio include polygyny with related queens, polyandry, and partial queen control of sex allocation (Bourke and Franks 1995). All these factors may work in concert.

The second implication is that the deviation from the expected values based on relatedness asymmetry and worker control should vary across species and populations whenever the cost of manipulation varies. With male elimination, for example, costs may vary according to the developmental stage at which workers can identify males and the error rate in identification. The cost of male elimination could also vary according to how efficiently the resources already invested in males can be recycled, for example, by feeding female larvae with the males eliminated. Finally, the cost of manipulation may vary depending on stochastic variations in resource abundance with, for instance, the cost of male elimination being greatly reduced or even absent when resource scarcity forces colonies to reduce the amount of sexual brood. When sex allocation is manipulated by directing a greater proportion of females toward queen development, costs are expected to vary depending on the relationship between the size of the worker force and colony productivity. Hence, the cost of sex ratio manipulation should vary across species according to differences in life history. This prediction is in line with the finding that there is great variation in the degree of female bias across monogynous species (reviewed in Boomsma 1989; Pamilo 1990).

Variance in manipulation cost could also arise from variation in resource abundance. The abundance of food in the habitat may determine to what extent colony productivity is limited by the number of eggs versus the amount of food available. When food is the limiting factor, the cost of sex ratio manipulation consists not so much in the destruction of brood but in the loss of resources invested in males prior to elimination. By contrast, when food is abundant and eggs are limiting, workers may possibly raise all individuals. In this situation, decreased investment in males cannot be redirected into additional investment into females, leading to greater cost of sex ratio manipulation. A counterintuitive prediction is thus that workers are more likely to benefit from sex ratio biasing when resource availability is low (i.e., when colonies are unlikely to be egg limited) than when resource availability is high (i.e., when colonies are more likely to be egg limited). Female bias in sex allocation should therefore be negatively correlated with resource abundance and overall productivity. Because this prediction holds at the population as well as at the colony level, it could be tested with data from studies manipulating colony food levels. So far, however, studies of this type have provided contradictory results. Food supplementation has caused an increased female bias among sexuals in some studies (Deslippe and Savolainen 1995; Herbers and Banschbach 1998; Morales and Heithaus 1998), no change in investment in others (Aron et al. 2001; DeHeer et al. 2001; Bono and Herbers 2003), and an increase in male production (in association with a host of changes in colony social structure and demography) in one study (Herbers and Banschbach 1999). The interactions between resource availability and colony investment are thus not yet well understood, making it difficult to draw conclusions about the relationship between resource abundance and costs of sex ratio manipulation.

Our model shows that the shape of the cost function has important implications about patterns of sex ratios at the colony level. While linear and accelerating costs lead to a single equilibrium, decelerating costs lead to sex ratio specialization with part of the colonies producing a secondary sex ratio identical to the primary sex ratio while the others raise only female sexuals. Sex ratio specialization (also termed "split sex ratios") has been observed in a large number of social Hymenoptera. Several explanations for split sex ratios have been proposed. One is that the marginal value of one sex relative to the other varies across colonies (Grafen 1986; Boomsma and Grafen 1990). In the presence of such variation, colonies would specialize in the sex providing the higher fitness return. Variation in relative marginal values of the sexes can arise in two ways. Grafen (1986) proposed that colonies could vary in the cost ratios of producing males and females, for example, because some colonies have preferential access to particular resources needed for the production of one sex (Grafen 1986; Boomsma 1993). This hypothesis can be tested with experiments involving food supplementation, with the caveat that it is often difficult to know what type of food may increase or decrease the relative cost of producing males versus females. The only study supplementing colonies with different food types did not detect any effect of food supplementation on colony investment (Bono and Herbers 2003). Currently it is therefore impossible to draw a conclusion about the validity of Grafen's hypothesis. Alternatively, Boomsma and Grafen (1990, 1991) proposed that the relative marginal values of males and females could vary between colonies because of differences in colony kin structure. If, for instance, queens vary in mating frequency, workers in colonies with aboveaverage relatedness asymmetry (i.e., below-average mating frequency) should preferentially produce females, whereas workers in other colonies should specialize in the pro- 
duction of males. Evidence for this hypothesis has been found in a number of species (e.g., Müller 1991; Queller et al. 1993; Sundström 1994; Sundström et al. 1996; Walin and Seppä 2001; Hammond et al. 2002). In others, however, sex ratio specialization occurred but was not associated with differences in relatedness asymmetry (e.g., Helms 1999; Brown and Keller 2000; Fjerdingstad et al. 2002). An alternative explanation for split sex ratios is provided by the constant male and the constant female hypotheses (Frank 1987a, 1987b). These hypotheses propose that split sex ratios could arise when one sex undergoes local competition (most likely local mate competition in males, local resource competition in females). Local competition leads to decelerating fitness returns with increasing investment in a sex. Frank therefore predicted that colonies should invest into the locally competing sex only up to a critical level where that sex provides the same fitness return as the other sex. Colonies that have fewer resources than the critical level should thus invest only in the competing sex while colonies with more resources should produce both sexes. Evidence for these two hypotheses has been found both in species with local mate competition and in those with local resource competition (reviewed in Bourke and Franks 1995). Other species, however, did not fit Frank's hypotheses despite the occurrence of local competition (Bourke and Franks 1995).

The reason underlying sex ratio specialization in our model differs qualitatively from those implied in the hypotheses outlined above. In these, polymorphism in investment is caused by selective pressures differing between colonies due to variation in habitat quality or colony kin structure. In our case, sex ratio specialization arises through a process of evolutionary branching during which individual strategies can evolve toward either of two equilibria ( $m_{\text {none }}$ or $m_{\text {all }}$ ), both of which offer equal fitness returns. This process does not rely on any intrinsic differences between colonies, and our model therefore predicts no association between a colony's sex allocation and its habitat quality or kin structure. But our model makes two other predictions that can be tested experimentally. The first is that colonies producing only females should have a lower overall productivity than colonies producing both sexes. This is because manipulation of sex ratio occurs only in exclusively female-producing colonies and lowers their productivity. The second prediction is that, across populations, the difference in productivity between colonies producing females and those producing both sexes should be negatively correlated with the female bias in population sex ratio and the frequency of female-producing colonies (cf. fig. 4).

In a recent article, Roisin and Aron (2004) predicted that sex ratio specialization could also evolve when queens control the number of female eggs produced and workers control the proportion of male and female eggs raised. In this situation, sex ratio specialization will occur because queens apply two strategies that have equal fitness payoffs. One strategy consists of strongly limiting the number of female eggs (resulting in workers raising only male sexuals) and the other of producing female eggs ad libitum (resulting in an all-female production). This suggests that there are different ways in which evolutionary branching of strategies can evolve under queenworker conflict, and thus there are several routes leading to sex ratio specialization.

Because the shape of the cost function has a major impact on the evolution of sex ratio manipulation, it is important to consider the biological mechanisms that could generate differently shaped costs functions. Accelerating costs imply that the decrease in productivity for a given change in sex ratio increases with overall manipulation. Such a relationship is expected to results whenever workers manipulate the sex ratio by increasing the proportion of females developing into queens. In several species, colony productivity has been shown to be a saturating function of worker number (e.g., Brian 1956; Tschinkel 1993; Sundström 1995). Increased allocation of females to queen development (and the concomitant decrease in worker allocation) thus leads to an accelerating reduction in colony productivity. Linear costs arise when the effect of a small change in sex ratio on productivity is constant and independent of the overall degree of manipulation. Linear costs could result from the replacement of male brood by female brood. The cost of eliminating one male larva (i.e., the amount of energy lost per eliminated male or the time spent by workers handling brood) is likely to be constant and independent of the total number of males eliminated in the colony. Finally, decelerating costs occur whenever the cost of a small change in sex ratio decreases with the overall degree of manipulation. An extreme kind of such a relationship can result if the overall cost of manipulation is fixed and identical for any sex ratio alteration $>0$. This would be the case if the loss of productivity would only stem from the time and energy that workers spend to keep track of the current brood sex ratio in order to adapt their manipulation behavior rather than the waste of energy invested in eliminated male larvae.

Currently no data are available on the relationship between mechanisms of sex ratio manipulation and the costs they imply. The above scenarios thus remain hypothetical. Empirical data on the shape of cost functions would be extremely useful. One way to get insight into costs of manipulation would be to experimentally alter the primary sex ratio of laboratory colonies. In this way, workers would be forced to manipulate brood more or less in order to attain their desired sex allocation. The costs of brood manipulation could be assessed by measuring the sexual pro- 
duction of colonies with different primary sex ratios. Potential sources for eggs with female-biased sex ratios are laboratory colonies in an early stage of colony development or colonies artificially shifted in season that produce worker-destined brood. Male eggs could be obtained from queenless colonies with worker reproduction. In all of these cases, controls would be necessary in order to disentangle the effects of altered costs of sex ratio manipulations from effects of nestmate recognition and rejection of foreign brood. It would also be interesting to determine the costs and benefits of male elimination directly. This could be done by transferring male larvae or pupae between colonies or removing them. If male larvae serve as a valuable food reserve, colonies supplied with additional brood should do better than colonies from which male brood has been removed.

In conclusion, our model shows that the costs associated with sex ratio manipulation influence sex allocation at both the colony and population levels. By constraining the workers' possibilities of altering the queen-controlled primary sex ratio, manipulation costs also have an important impact on the resolution of sex ratio conflict. High costs can hinder workers from manipulating sex allocation and thus prevent the occurrence of actual conflict. Experiments addressing not only the benefits but also the costs of brood manipulation should allow us to gain insight in the mechanisms of sex ratio adjustment and more generally the outcome of queen-worker conflict in colonies of eusocial Hymenoptera.

\section{Acknowledgments}

We thank D. Sumpter for discussions on the model and K. Boomsma, A. Bourke, D. Ebert, P. Pamilo, and members of the Keller lab for helpful comments on the manuscript. This work was supported by grants from the Swiss National Science Foundation.

\section{Literature Cited}

Aron, S., L. Keller, and L. Passera. 2001. Role of resource availability on sex, caste and reproductive allocation ratios in the Argentine ant Linepithema humile. Journal of Animal Ecology 70:831-839.

Bono, J. M., and J. M. Herbers. 2003. Proximate and ultimate control of sex ratios in Myrmica brevispinosa colonies. Proceedings of the Royal Society of London B 270:811-817.

Boomsma, J. J. 1989. Sex-investment ratios in ants: has female bias been systematically overestimated? American Naturalist 133:517-532.

- 1993. Sex ratio variation in polygynous ants. Pages 86-109 in L. Keller, ed. Queen number and sociality in insects. Oxford University Press, Oxford.
Boomsma, J. J., and A. Grafen. 1990. Intraspecific variation in ant sex ratios and the Trivers-Hare hypothesis. Evolution 44:1026-1034.

1991. Colony-level sex ratio selection in the eusocial Hymenoptera. Journal of Evolutionary Biology 4: 383-407.

Bourke, A. F. G., and N. R. Franks. 1995. Social evolution in ants. Princeton University Press, Princeton, N.J.

Brian, M. V. 1956. Group form and the cause of working inefficiency in the ant Myrmica rubra L. Physiological Zoology 29:173-194.

Brown, W. D., and L. Keller. 2000. Colony sex ratios vary with queen number but not relatedness asymmetry in the ant Formica exsecta. Proceedings of the Royal Society of London B 267:1751-1757.

Chapuisat, M., L. Sundström, and L. Keller. 1997. Sexratio regulation: the economics of fratricide in ants. Proceedings of the Royal Society of London B 264:12551260.

Christiansen, F. B. 1991. On conditions for evolutionary stability for a continuously varying character. American Naturalist 138:37-50.

Crozier, R. H., and P. Pamilo. 1996. Evolution of social insect colonies: sex allocation and kin-selection. Oxford University Press, Oxford.

DeHeer, C. J., V. L. Backus, and J. M. Herbers. 2001. Sociogenetic responses to ecological variation in the ant Myrmica punctiventris are context dependent. Behavioral Ecology and Sociobiology 49:375-386.

Deslippe, R. J., and R. Savolainen. 1995. Sex investment in a social insect: the proximate role of food. Ecology 76:375-382.

Eshel, I., and U. Motro. 1981. Kin selection and strong evolutionary stability of mutual help. Theoretical Population Biology 19:420-433.

Fjerdingstad, E. J., P. J. Gertsch, and L. Keller. 2002. Why do some social insect queens mate with several males? testing the sex-ratio manipulation hypothesis in Lasius niger. Evolution 56:553-562.

Frank, S. A. 1987a. Individual and population sex allocation patterns. Theoretical Population Biology 31:4774 .

- 1987b. Variable sex ratio among colonies of ants. Behavioral Ecology and Sociobiology 20:195-201.

Geritz, S. A. H., E. Kisdi, G. Meszena, and J. A. J. Metz. 1998. Evolutionarily singular strategies and the adaptive growth and branching of the evolutionary tree. Evolutionary Ecology 12:35-57.

Grafen, A. 1986. Split sex ratio and the evolutionary origin of eusociality. Journal of Theoretical Biology 122:95121.

Hammond, R. L., M. W. Bruford, and A. F. G. Bourke. 2002. Ant workers selfishly bias sex ratios by manipu- 
lating female development. Proceedings of the Royal Society of London B 269:173-178.

Helms, K. R. 1999. Colony sex ratios, conflict between queens and workers, and apparent queen control in the ant Pheidole desertorum. Evolution 53:1470-1478.

Herbers, J. M., and V. S. Banschbach. 1998. Food supply and reproductive allocation in forest ants: repeated experiments give different results. Oikos 83:145-151.

. 1999. Plasticity of social organization in a forest ant species. Behavioral Ecology and Sociobiology 45: 451-465.

Morales, M., and E. Heithaus. 1998. Food from seeddispersal mutualism shifts sex ratios in colonies of the ant Aphaenogaster rudis. Ecology 79:734-739.

Müller, U. G. 1991. Haplodiploidy and the evolution of facultative sex-ratios in a primitively eusocial bee. Science 254:442-444.

Pamilo, P. 1990. Sex allocation and queen-worker conflict in polygynous ants. Behavioral Ecology and Sociobiology $27: 31-36$.

- 1991. Evolution of colony characteristics in social insects. I. Sex allocation. American Naturalist 137:83107.

Queller, D. C., J. E. Strassmann, C. R. Solis, C. R. Hughes, and D. M. Deloach. 1993. A selfish strategy of social insect workers that promotes social cohesion. Nature 365:639-641.

Reuter, M., and L. Keller. 2001. Sex ratio conflict and worker production in eusocial Hymenoptera. American Naturalist 158:166-177.

Roisin, Y., and S. Aron. 2004. Split sex ratios in perennial social Hymenoptera: a mixed evolutionary stable strategy from the queens' perspective? American Naturalist 162:624-637.

Sundström, L. 1994. Sex ratio bias, relatedness asymmetry and queen mating frequency in ants. Nature 367:266268.

1995. Sex allocation and colony maintenance in monogyne and polygyne colonies of Formica truncorum (Hymenoptera: Formicidae): the impact of kinship and mating structure. American Naturalist 146:182-201.

Sundström, L., M. Chapuisat, and L. Keller. 1996. Conditional manipulation of sex ratios by ant workers: a test of kin selection theory. Science 274:993-995.

Taylor, P. D. 1996. Inclusive fitness arguments in genetic models of behaviour. Journal of Mathematical Biology 34:654-674.

Trivers, R. L., and H. Hare. 1976. Haplodiploidy and the evolution of the social insects. Science 191:249-263.

Tschinkel, W. R. 1993. Sociometry and sociogenesis of colonies of the fire ant Solenopsis invicta during one annual cycle. Ecological Monographs 63:427-457.

Walin, L., and P. Seppä. 2001. Resource allocation in the red ant Myrmica ruginodis: an interplay of genetics and ecology. Journal of Evolutionary Biology 14:694-707.

Associate Editor: Peter Nonacs 